\title{
Hall Effect in Quasi One-Dimensional Organic Conductors
}

\author{
Gladys León and Thierry Giamarchi
}

University of Geneva, 24 Quai Ernest-Ansermet $\mathrm{CH}-1211$ Geneva 4, Switzerland

We study the Hall effect in a system of weakly coupled Luttinger Liquid chains, using a Memory function approach to compute the Hall constant in the presence of umklapp scattering along the chains. In this approximation, the Hall constant decomposes into two terms: a high-frequency term and a Memory function term. For the case of zero umklapp scattering, where the Memory function vanishes, the Hall constant is simply the band value, in agreement with former results in a similar model with no dissipation along the chains. With umklapp scattering along the chains, we find a power-law temperature dependance of the Hall constant. We discuss the applications to quasi $1 D$ organic conductors at high temperatures.

PACS numbers: 71.10.Pm, 71.10.Hf, 71.27.+a, 72.80.-r, 72.80.Le

\section{INTRODUCTION}

Within the organic conductors, the Bechgaard salts $(T M T S F)_{2} X$, have been widely studied for their many interesting properties and their complex phase diagram. ${ }^{1}$ In particular their low dimensionality makes them very special: they experience a dimensional crossover from a 1D Luttinger Liquid ${ }^{2}$ (LL) to a higher dimensional metallic state ${ }^{3}$. An important open question is the Hall effect in such an interacting system made of coupled chains. At low temperature theories based on a Fermi liquid description are possible ${ }^{4}$. In the high temperature coupled LL regime a description of the Hall effect was only possible in the quite restricted case where no scattering exists inside the chains ${ }^{5}$. In that case one surprisingly obtains a temperature independent Hall resistance given by the simple band value even if interactions are present in the system. Up to now, no theory when scattering is present in the chains was available. Measurements ${ }^{6,7}$ were made in the compound $(T M T S F){ }_{2} P F_{6}$ giving different results for the Hall constant above the dimensional crossover 
temperature and making the need for a theoretical description even more important. We present here such a theory of the Hall effect of coupled LL. We use a Memory matrix approach to compute the Hall constant and find out its temperature dependance.

\section{MODEL}

We consider a model of coupled LL chains (along the $\mathrm{x}$-axis) with umklapp scattering, coupled by a transverse hopping (y-axis) and a magnetic field applied perpendicular to the plane of the chains (z-axis). The hamiltonian $\hat{H}=\hat{H}_{0}+\hat{H}_{1}$ for fermions with spins, decomposes into a unperturbed part $\hat{H}_{0}$ and a perturbation term $\hat{H}_{1}$. The first part is:

$$
\begin{aligned}
\hat{H}_{0}=\int d x\left[\sum_{i, \sigma} v_{F} \hat{\psi}_{i, \sigma}^{\dagger}\right. & \tau_{3}\left(-i \partial_{x}\right) \hat{\psi}_{i, \sigma}-\alpha \sum_{i, \sigma} \hat{\psi}_{i, \sigma}^{\dagger} \partial_{x}^{2} \hat{\psi}_{i, \sigma} \\
& \left.+U \sum_{i, \sigma, \sigma^{\prime}} \rho_{i, \sigma} \rho_{i, \sigma^{\prime}}-t_{\perp} \sum_{\langle i, j\rangle, \sigma} \hat{\psi}_{i, \sigma}^{\dagger} \hat{\psi}_{j, \sigma} e^{-i \frac{e}{c} A_{i, j}}\right],
\end{aligned}
$$

where $\hat{\psi}$ is a two component vector composed from the right and left-moving electrons, $\tau_{3}$ is a pauli matrix and $A_{i, j}=\int_{i}^{j} \mathbf{A} d \mathbf{l}$, where we use the Landau gauge $A_{y}=H x$. The second term in (1) is a nonlinear correction to the spectrum. Including such a band curvature term is necessary due to the fact that in chains with linear spectrum, the particle-hole symmetry gives a zero Hall resistivity. The spectrum is thus $\epsilon_{ \pm}= \pm v_{F}\left(p \mp p_{F}\right)+\alpha\left(p \mp p_{F}\right)^{2}$. The third term in (1) are the momentum conserving interaction processes ${ }^{8}$. The last term is the coupling between the chains. The interaction term $\hat{H}_{1}$ contains the umklapp processes, where two particles are scattered from one side to the other side of the Fermi surface, transferring or absorbing momentum from the lattice. For simplicity we consider here the case of halffilled commensurate system which satisfy $4 k_{F}=\frac{2 \pi}{a}$, where $a$ is the lattice spacing along the chains. Then $\hat{H}_{1}$ is

$$
\hat{H}_{1}=g_{3} \int d x \sum_{i, \sigma}\left(\hat{\psi}_{R, i, \sigma}^{\dagger} \hat{\psi}_{R, i,-\sigma}^{\dagger} \hat{\psi}_{L, i,-\sigma} \hat{\psi}_{L, i, \sigma}+\text { h.c. }\right)
$$

To compute the Hall constant $R_{H}$ we use a Memory function approach, which consists on a perturbative expansion of the resistivities ${ }^{9}$. Within this approach an expression of $R_{H}$ was obtained ${ }^{10}$ for the isotropic Hubbard model. In the case of coupled chain the corresponding expression correctly 
taking the anisotropy into account becomes ${ }^{11}$

$$
R_{H}(z)=\frac{1}{i \chi_{y}^{0}} \lim _{H \rightarrow 0} \frac{\Omega_{x y}+i M_{x y}(z)}{H}
$$

where $\Omega_{x y}$, the frequency matrix, is defined as $\equiv \frac{1}{\chi_{x}^{0}}\left\langle\left[\hat{J}_{x}, \hat{J}_{y}\right]\right\rangle$. This term represents the high-frequency limit and when there is no perturbation (umklapp scattering) it gives back the exact result of Ref 5 . This check ensures that the Memory function approximation indeed captures the correct physics. The $\chi_{\nu}^{0}$ are the diamagnetic terms in the $x$ and $y$ directions. The second term in (3) is the Memory function, which is given by

$$
i \chi_{x}^{0} M(z)=-\frac{\left\langle\left\langle\hat{K}_{x} ; \hat{K}_{y}\right\rangle\right\rangle_{z}^{0}}{z}
$$

The $\hat{K}_{\nu}$ operators are the commutator between the umklapp term and the current along the $\nu$ direction $\hat{K}_{\nu}=\left[\hat{H}_{1}, \hat{J}_{\nu}\right]$.

\section{RESULTS}

The difficult part is to compute the correlation function $\left\langle\left\langle\hat{K}_{x} ; \hat{K}_{y}\right\rangle\right\rangle_{z}^{0}$. Technical details will be presented elsewhere ${ }^{11}$ and we quote here only the results. For weakly coupled 1/2-filled LL chains, the Hall constant has a power law dependance on temperature

$$
R_{H}=R_{H}^{0}\left[1-A\left(\frac{g_{3}}{u_{\rho}}\right)^{2}\left(\frac{\alpha T}{u_{\rho}}\right)^{3 K_{\rho}-3}\right]
$$

where $R_{H}^{0}$ is the band value ${ }^{5}, g_{3}$ is the umklapp parameter, $u_{\rho}$ the velocity of the charge excitations along the chains and $A$ a constant. The LL parameter ${ }^{8}$ $K_{\rho}$ controls the temperature dependence (we take $K_{\sigma}=1$ for the spin part). When there are no interactions $K_{\rho}=1$ giving no temperature dependence for $R_{H}$. For repulsive interactions $K_{\rho}<1$ (as in the organic conductors ${ }^{2}$ ). In this case two important limits appears: for small $g_{3}$ or large temperature, $R_{H} \rightarrow R_{H}^{0}$. Note that the power law dependence found for the Hall constant is different from the the one found for the conductivity along LL chains with umklapp scattering ${ }^{12} \sigma(T) \propto T^{3-4 K_{\rho}} / g_{3}^{2}$.

\section{DISCUSSION AND CONCLUSIONS}

Using a Memory function approach, we obtained the Hall constant in weakly coupled LL chains with umklapp scattering along the chains, and we 
obtained a power law dependance for $1 / 2$-filled commensurate systems. It would be interesting to compare such result with measurements of the Hall resistance in purely half filled organic compounds. To compare with the Bechgaard salts it is important to take into account that these compounds have both half and quarter filled commensurate character. If quarter filling umklapp $g_{3,1 / 4}$ is present one expects the Hall resistance to be

$$
\frac{R_{H}}{R_{H}^{0}}=1-A\left(g_{3,1 / 2}\right)^{2} T^{\mu}+B\left(g_{3,1 / 4}\right)^{2} T^{\nu} .
$$

where the calculation for $\nu$ will be presented elsewhere. ${ }^{11}$ Longitudinal transport is dominated ${ }^{2}$ by the quarter filled commensurability and it will be interesting to check whether the same is true for the Hall resistance. To decide on that point it will however be useful to have additional measurements of Hall resistance in order to reconcile the two different temperature behaviors that are for now observed experimentally in the same compound ${ }^{6,7}$.

\section{ACKNOWLEDGEMENTS}

This work has been supported in part by the Swiss National Fund for research under MANEP and Division II.

\section{REFERENCES}

1. D. Jérome, Chem. Rev. 104, 5565 (2004).

2. A. Schwartz, M. Dressel, G. Grüner, V. Vescoli, L. Degiorgi, and T. Giamarchi, Phys. Rev. B 58, 1261 (1998).

3. T. Giamarchi, Chem. Rev. 104, 5037 (2004).

4. V. M. Yakovenko and A. T. Zheleznyak, Synth. Met. 120, 1083 (2001).

5. A. Lopatin, A. Georges, and T. Giamarchi, Phys. Rev. B 63, 075109 (2001).

6. J. Moser, J. R. Cooper, D. Jérome, B. Alavi, S. Brown, and K. Bechgaard, Phys. Rev. Lett. 84, 2674 (2000).

7. G. Mihaly, I. Kezsmarsky, F. Zamborsky, and L. Forro, Phys. Rev. Lett. 84, $2670(2000)$.

8. T. Giamarchi, Quantum Physics in One Dimension (Oxford University Press, Oxford, 2004).

9. E. Fick and G. Sauermann, The Quantum Statistics of Dynamic Processes (Springer, Berlin, 1990).

10. E. Lange, Phys. Rev. B 6, 3907 (1997).

11. G. León and T. Giamarchi, 2005, in preparation.

12. T. Giamarchi, Phys. Rev. B 44, 2905 (1991). 